\title{
A Review of Spectrum Sensing Techniques for Cognitive Radio
}

\author{
Md. Shahnawaz Shaikh \\ AITR \\ Indore
}

\author{
Kamlesh Gupta \\ AITR \\ Indore
}

\begin{abstract}
This paper explores the concept for dynamic spectrum allocation and investigates different aspects of spectrum sensing in cognitive radio (CR) technology. CR systems are radios with the ability to exploit their environment to increase spectral efficiency and capacity. CR technology explores, an opportunistic and promising technology to utilize available communication spectrum in efficient and dynamic way. It proposes secondary users to optimally utilize reserved frequency bands of primary users that are not occupied all the time by primary users. This increases spectrum efficiency of available communication spectra. Spectrum sensing is a basic approach and requirement to implement cognitive radio technology. It is one of the most challenging issues of CR process to prevent interference between primary user and secondary user. In this paper we explore various spectrum sensing techniques such as matched filtering, energy detection, cyclostationary feature detection, and cooperative spectrum sensing techniques. We also highlight the strengths, weaknesses, parameters concerned and feasibilities of these techniques with comparison among them. Various challenges and the parameters which can affect performance of these techniques are also discussed.
\end{abstract}

\section{Keywords:}

cognitive, spectrum sensing, energy detection, matched filtering, cyclostationary detection, cooperative sensing.

\section{INTRODUCTION}

Cognitive radio (CR) concept can be applied to many advanced and challenging communication as well as networking systems. The word cognitive means, pertained to cognition or the action or process of knowing. It also means, the mental process of getting knowledge through thought, experience and the senses. Thus, in communication systems CR defines the radio with ability to sense reserved, idle communication spectra which can be utilized by secondary users for other applications during its idle period [5], [6]. CR maximizes throughput of spectrum to increase spectrum efficiency and facilitate interoperability by providing access to secondary user group for other applications [7]. Cognitive radio concept may be applicable at 400-800 MHz (UHF TV bands) and 3-10 $\mathrm{GHz}$. Moreover, for long range communication applications these frequency bands have good propagation properties. Cognitive radio systems offer opportunity to use dynamic spectrum management techniques to immediately utilize available local spectrum.
White spaces, gray spaces and black spaces recognize the occupancy of spectrum with noise to define interference level. White spaces: free from interference due to other RF bands, excepting noise due to natural or artificial sources. Gray spaces: partially affected by interference and noise.

Black spaces: completely occupied by interference and noise. Figure 1 shows CR environment which exhibits primary and secondary user terminal pair, serving $\mathrm{CR}$ technology by $\mathrm{CR}$ sensor. A CR sensor has prior knowledge about signal threshold energy level, frequency band and cyclostationary features of signal to perform useful sensing. Sensing performance of CR sensors may get affected due to noise and shadowing which may cause false alarm and missed-detection.

This paper is organized as follows: Section II defines categorization of signal processing techniques for spectrum sensing. Section III presents most popular non-cooperative spectrum sensing techniques. In this section spectrum sensing techniques are explained with their relative features and limitations. Cooperative sensing and its types are defined in section IV. Section V gives process flow chart of non cooperative techniques and Section VI concludes this paper.

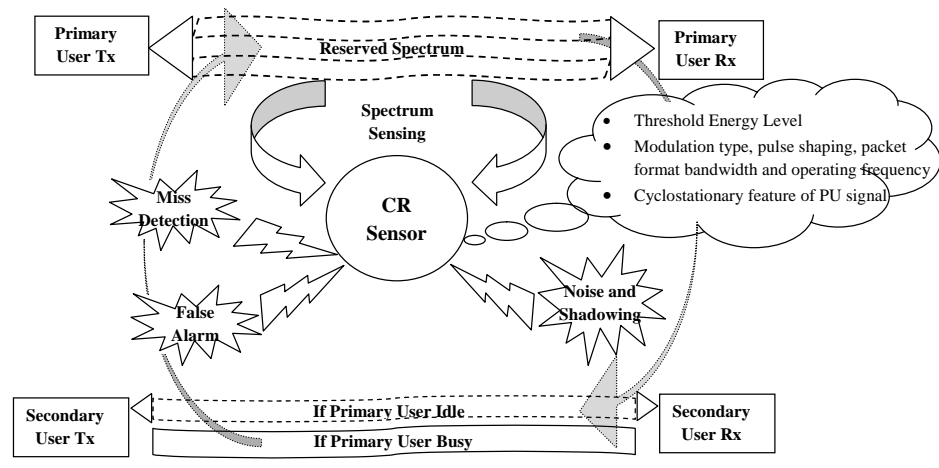

Fig. 1. Cognitive Environment

\section{SPECTRUM SENSING TECHNIQUES}

Classification of most popular spectrum sensing techniques for implementation of CR technology is shown in Figure 2. This figure 
also categorizes non-cooperative and cooperative spectrum sensing techniques.

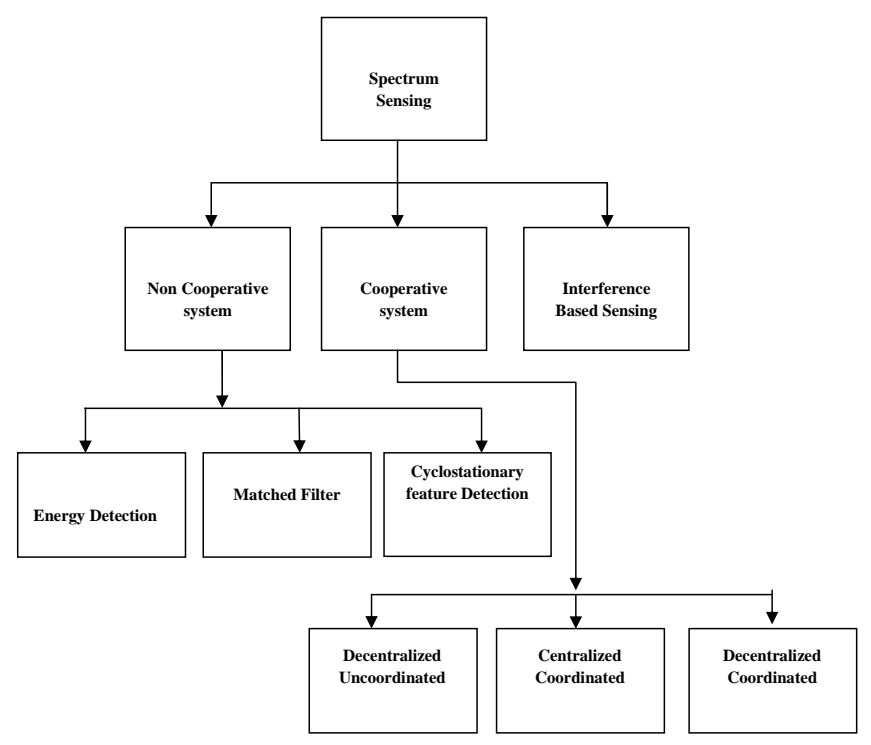

Fig. 2. Classification of spectrum sensing techniques [9].

\section{NON-COOPERATIVE SENSING}

Non-cooperative sensing techniques need detection of primary user signal by some parameter measurement and filtering. The location of primary user is not known. It is based on following hypotheses

$$
X(t)= \begin{cases}n(t) & H_{0} \\ s(t)+n(t) & H_{1}\end{cases}
$$

In above equation $H_{0}$ denotes absence of primary user and $H_{1}$ shows its presence. $n(t)$ and $s(t)$ represents noise and primary user massage signal respectively. Popular non-cooperative Sensing techniques are:

-Matched filtering.

-Energy detection.

- Cyclostationary feature detection.

\subsection{Matched Filtering:}

Matched filter detection is very accurate and most promising technique for spectrum sensing which maximizes SNR. A matched filter is a linear filter, based on coherent detection of primary user signal [1], [2], [3], [5]. When we pass the signal from the filter, it passes the useful signal while attenuate the noise signal at the same time. If the desired signal is present, large peak appears at the output of the filter, otherwise the signal is not present (only noise is there) [19].

Primary user information e.g., modulation type and order, pulse shaping, packet format bandwidth and operating frequency might be pre-stored in CR memory. The cyclostationary feature detector performs, timing and carrier synchronization as well as channel equalization for received signal[1], [2], [3], [6], [7]. In matched filter operation, received signal is convolved with the filter impulse response, which is time shifted and mirror version of reference signal [1], [2], [5],|8]. Optimal detector in stationary Gaussian noise used as matched filter when secondary user has knowledge about information of primary user signal [8], [10]. Matched filter performs poorly in case of incomplete or inaccurate information [8], [10]. The operation of matched filter detection is expressed as [1], [2], [3], [8].

$$
Y[n]=\sum_{k=-\infty}^{+\infty} h[n-k] z[k]
$$

Where $z$ is unknown signal and is convolved with the $h$, the impulse response of matched filter that is matched to the reference signal for maximizing the SNR [1], [2], [3], [8].

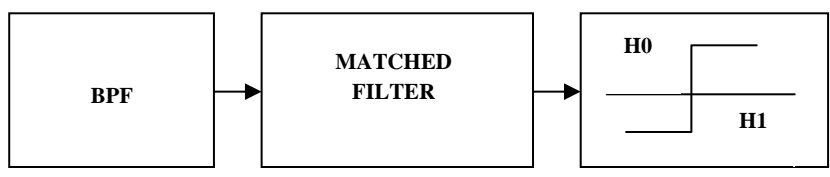

Fig. 3. Matched Filtering [2].

\section{Features:}

(1) As Cognitive Radio user knows information of the licensed user signal, matched filter detection requires less detection time due to high processing gain [1], [2], [3], [6], [7], [8].

(2) Matched filtering needs short time to achieve a certain probability of false alarm or probability of missed-detection [2], [7].

\section{Limitations:}

There are some limitations of this technique which are:

(1) It requires a prior knowledge of every primary signal [1], [2], [3], [6], [7], [8].

(2) CR needs a dedicated receiver for every type of primary user [1], [3], [6].

(3) Cognitive radio needs receivers for all signal types, the implementation complexity of sensing unit is impractically large [2], [7].

(4) Matched filtering consume large power as various receiver algorithms need to be executed for detection [2], [7]

\subsection{Energy Detection}

Energy detection is the non-coherent detection based sensing technique of CR [6]. The energy detection method is very simple to implement as compared to other techniques, since it does not require the prior knowledge about the structure or format of the primary user signal. The energy detection method calculates energy of the signal of desired frequency band and compares it with a predecided threshold energy level [16]. The signal is said to be present at a particular frequency band, if energy of the signal exceeds energy level of the decision threshold [4]. Otherwise processed frequency band is expected to be idle and could be accessed by CR.

$$
X(t)= \begin{cases}n(t) & H_{0} \\ s(t)+n(t) & H_{1}\end{cases}
$$

where $s(t)$ denotes primary user signal while $n(t)$ is noise signal received with primary user signal. $X(t)$ represents signal received 
$H_{0}$ represents presence of noise only, while $H_{1}$ represents primary user signal is present [16], [17].

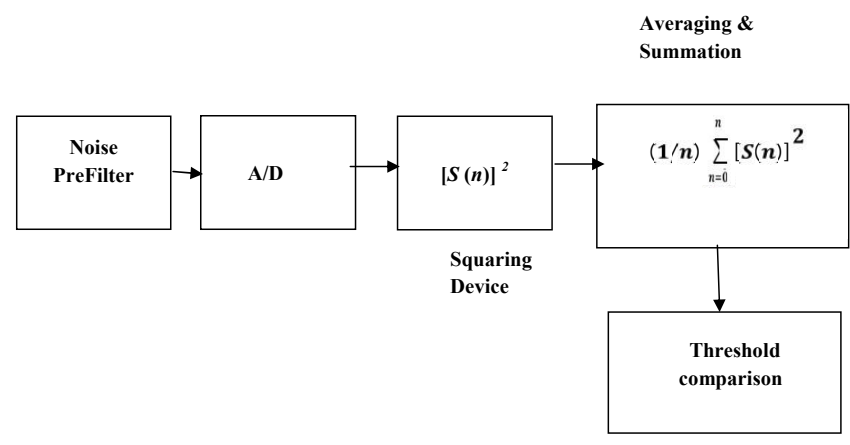

Fig. 4. Energy Detection Process [13 $14,15,16,17,18]$.

Figure 4 shows block diagram of energy detector. It consist of noise pre-filter, which removes noise from received signal, to be converted in digital signal. Square law device squares the digital signal to calculate power within specified window. Averaging and summation is performed to calculate energy over FFT window for duration $T$ [13, 14, 15, 16, 17, 18].

\section{Features:}

(1) Energy detection method is popular due to its simplicity, ease of implementation and applicability [16].

(2) It has low computational and implementation cost [16].

\section{Limitations:}

There are several limitations of energy detectors that might diminish their simplicity in implementation [23].

(1) Threshold used for primary user detection is highly susceptible to changing noise levels. Presence of any undesired band possessing equal energy level might confuse the energy detector [16].

(2) Energy detector does not differentiate between primary user (PU) signals, noise and interference. So it cannot be beneficial to prevent interference [6].

(3) Energy detector is not useful for direct sequence, frequency hopping signals and spread spectrum signals [6].

(4) When there is heavy fluctuation in signal power so it becomes difficult to differentiate the desired signal [6].

(5) Compared to matched filter detection, energy detection technique requires longer time to achieve desired performance level [16].

\subsection{Cyclostationary Feature Detection}

Cyclostationary feature detection (CFD) is the two dimensional complex valued spectrum sensing technique. Modulated primary user (PU) signals associated with sine wave carriers, pulse trains, hoping sequences or cyclic prefixes exploit periodicity. The mean and autocorrelation of PU signals exhibit periodicity which is typically introduced in signal format. This periodicity characterizes cyclostationary feature of PU signals [1, 2 , 3 , 4 , 5 , 5 , 6 , 7], [13]. Receiver exploits cyclostationary features of PU signals such as carrier phase, pulse timing, or direction of arrival. These parameters are used for detection of a random signal in radio environment in presence of other modulated signals and noise [3], [6], [7], [13].
Unlike common analysis as in energy detection and matched filtering, stationary random signals are based on autocorrelation functions and power spectral density [6]. Cyclostationary signal exhibits correlation between widely separated spectral components due to spectral redundancy caused by periodicity [6].

The received signal is assumed to be of following simple form

$$
X(n)=s(n)+w(n)
$$

The cyclic spectral density (CSD) function of a received signal can be calculated as

$$
S(f, \alpha)=\sum_{\tau=-\infty}^{\infty}\left(R_{Y}^{\alpha}\right)(T) e^{-2 j \pi f T}
$$

Where

$$
\left(R_{Y}^{\alpha}\right)(T)=E\left[y(n+\tau) y(n-\tau) e^{2 j \pi \alpha n}\right]
$$

is the cyclic autocorrelation function (CAF), $\alpha$ is the cyclic frequency and $\tau$ is time delay associated with CAF over time interval $T$. When the cyclic frequency is equal to the fundamental frequency $f$ of transmitted signal $X(n)$, the CSD function has its maximum values [2], [3], [6], [7]. Cyclic frequencies are used as features for identifying transmitted PU signals. It can be assumed to be known or they can be extracted [7]. Spectral correlation function is also termed as cyclic spectrum [3], [7]. Signal analysis in cyclic spectrum domain preserves phase and frequency information related to timing parameters in modulated signals. As a result, overlapping features in the power spectrum density are non-overlapping features in the cyclic spectrum [6].

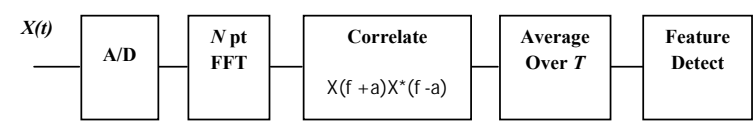

Fig. 5. Implementation of a cyclostationary feature detector

\section{Features:}

(1) Cyclostationary feature detection performs better than Energy detection technique in low SNR regions [2], [3], [8].

(2) It is not affected by noise uncertainties. It is robust to noise [2], [3], [8].

(3) Frequency and phase synchronization of signal is not required [8], [12].

\section{Limitations:}

(1) CFD requires long observation time, high sampling rate and higher computational complexity [2].

(2) CFD also requires the prior knowledge of primary user signal [2].

(3) There are possibilities of sampling time error.

\section{COOPERATIVE SENSING}

Detection of primary user signal may be affected by multipath fading, shadowing, and building penetration, which may cause incorrect or wrong sensing and judgment. It requires higher detection sensitivity to minimize the effect of uncertainty introduced by channel randomness and multipath fading [3], [12]. Increasing the detection sensitivity of CR approaches to techniques where more than 
one CR could work together and can share their sensing information with one another and cooperatively take decision about spectrum occupancy, introduces the notion of Cooperative or Collaborative or Distributed technique of spectrum sensing [12].

\subsection{Decentralized Uncoordinated Techniques}

Each CR user performs channel detection independently and does not support or share its information with other CR users [3].

\subsection{Centralized Coordinated Techniques:}

This technique designates a CR controller which is called fusion center (FC). It is in strong connectivity with its nearby CR users in its range. FC selects desired frequency band and inform all CR in network to perform local sensing. CR user in network detects idle channel or primary user; inform to CR controller which shares this information with all other CR users in network [3], [6], [10].

\subsection{Decentralized Coordinated Techniques:}

It does not require any CR controller or FC. Each CR user works as FC in network and provides coordination to other CR users [3], [10]. It is also called distributed cooperative sensing technique.

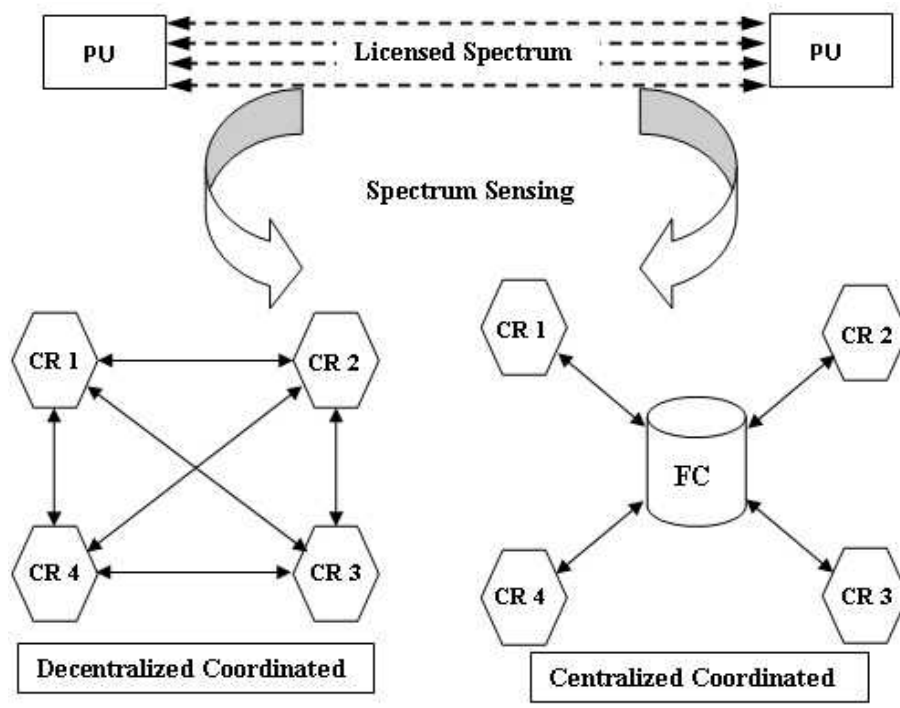

Fig. 6. Cooperative Spectrum Sensing Techniques

Figure 6 shows graphical representation of decentralized and centralized coordinated methods of cooperative spectrum sensing. In coordinated cooperative technique fusion center (FC) is coordinating with $4 \mathrm{CR}$ sensors while in uncoordinated technique all $4 \mathrm{CR}$ sensors are connected with all the other CRs in network without existence of central fusion center.

\section{Features:}

(1) Cooperative sensing decreases missed-detection and false alarm probabilities [2].

(2) It solves hidden primary user problem and also decreases sensing problems [2].

(3) It provides higher spectrum capacity gains than local sensing [2].
(4) It requires less sensitive detectors, which result in flexibilities, reduced hardware cost and complexities [12].

\section{Limitations:}

(1) Combining sensing results of more than one CR users having different sensitivities, is a difficult task [6].

(2) This technique requires a control channel to convey information among all CR users [6].

\section{FLOW CHART}

Implementation methodology of matched filtering, energy detection and cyclostationary feature detection techniques of spectrum sensing for cognitive radio is shown in Figure 7.

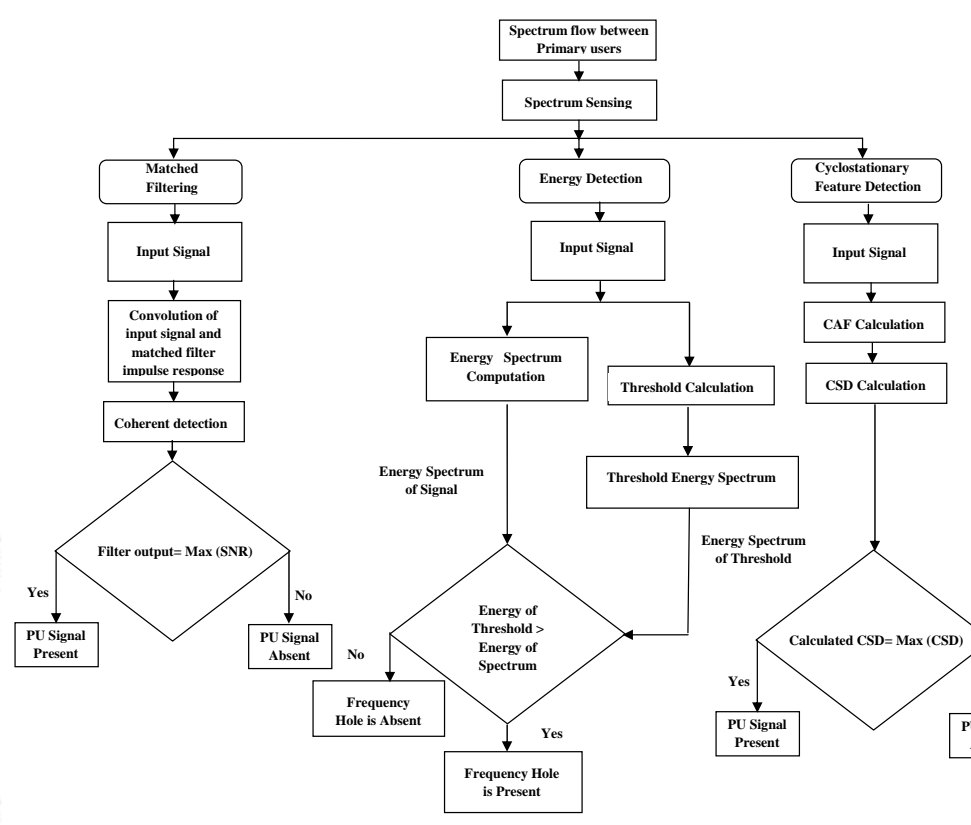

Fig. 7. Process Flow Chart of Spectrum Sensing Methods

\section{CONCLUSION}

Research on communication engineering is approaching is towards apex as the growing demand of wireless applications, especially for cellular and mobile communication has put a lot of constraints on the usage of available radio spectra and resources. Increasing demand of bandwidth for effective and healthy communication requires efficient and beneficial advancement with valuable spectrum utilization of communication resources. A novel approach to fulfill these requirements is to use CR concept. Most popular techniques for spectrum sensing are reviewed in this paper. CR systems increase throughput and user mobility over available spectra, which increase efficiency of communication networks. Cooperative spectrum sensing raises the strength of cognitive radio network by combining efforts of multiple cognitive sensors. 


\section{REFERENCES}

[1] Sajjad Ahmad Ghauri, I M Qureshi, M. Farhan Sohail, SherazAlam, M. Anas Ashraf, "Spectrum sensing for cognitive radio networks over fading channels," International Journal of Computer and Electronics Research Vol. 2, Issue 1, February 2013.

[2] Anita Garhwal and Partha Pratim Bhattacharya, "A survey on spectrum sensing techniques in cognitive radio," International Journal of Computer Science Communication Networks, Vol 1(2), 196-206.

[3] V. Stoianovici, V. Popescu, M. Murroni, "A survey on spectrum sensing techniques for cognitive radio," Bulletin of the Transilvania University of Brasov Vol. 15 (50) 2008.

[4] Anirudh M. Rao, B. R. Karthikeyan, Dipayan mazumdar, Govind R. Kadambi,"Energy detection technique for spectrum sensing in cognitive radio," SASTECH Vol. 9, Issue 1, April 2010.

[5] Mahmood A. Abdulsattar and Zahir A. Hussein, "Energy detection technique for Spectrum sensing in cognitive Radio," International Journal of Computer Networks Communications (IJCNC) Vol.4, No.5, September 2012.

[6] Danijela Cabric, Shridhar Mubaraq Mishra, Robert W. Brodersen Berkeley Wireless Research Center, University of California, Berkeley IEEE Paper, "Implementation issues in spectrum sensing for cognitive radios," in Proc. the 38th. Asilomar Conference on Signals, Systems and Computers, year 2004, pages 772-776.

[7] Tevfik Yucek and Huseyin Arslan, "A survey of spectrum sensing algorithms for cognitive radio applications," IEEE communications surveys tutorials, Vol. 11, no. 1, first quarter 2009.

[8] Mansi Subhedar1 and Gajanan Birajdar, "Spectrum sensing techniques in cognitive radio networks: a survey," International Journal of Next-Generation Networks (IJNGN) Vol.3, No.2, June 2011, DOI : 10.5121/ijngn.2011.3203 37.

[9] A. Rahim Biswas, Tuncer Can Aysal, Sithamparanathan Kandeepan, Dzmitry Kliazovich, Radoslaw Piesiewicz, "Cooperative shared spectrum sensing for dynamic cognitive radio networks," Broadband and Wireless Group, Create-Net International Research Centre, Trento, Italy, EUWB (FP7-ICT215669).

[10] Nishant Dev Khaira, Prateek Bhadauria, "Cooperative spectrum sensing and detection efficiency in cognitive radio network," International Journal of Electronics and Computer Science Engineering ISSN-2277-1956.

[11] Amir Ghasemi, Communications Research Centre Canada and University of Toronto Elvino S. Sousa, University of Toronto, "Spectrum sensing in cognitive radio networks: Requirements, Challenges and Design Trade-offs," IEEE Communications Magazine April 2008.

[12] Paul D. Sutton, Member IEEE, Keith E. Nolan, Member IEEE and Linda E. Doyle, Member IEEE, "Cyclostationary signatures in practical cognitive radio applications," IEEE journal on selected areas in communications, Vol. 26, no. 1, January 2008.

[13] Dong-Chan Oh and Yong-Hwan Lee, "Energy detection based spectrum sensing for sensing error minimization in cognitive radio networks," International Journal of Communication Networks and Information Security (IJCNIS) Vol. 1, No. 1, April 2009.
[14] Komal Arora, Ankush Kansal, Kulbir Singh, "Comparison of energy detection based spectrum sensing methods over fading channels in cognitive radio signal processing," An International Journal (SPIJ), Vol (5) : Issue (2) : 201144.

[15] Md. Shamim Hossain, Md. Ibrahim Abdullah, Mohammad Alamgir Hossain, "Energy detection performance of spectrum sensing in cognitive radio," I.J. Information Technology and Computer Science, 2012, 11, 11-17 Published Online October 2012 in MECS (http://www.mecs-press.org/) DOI: 10.5815/ijitcs.2012.11.02.

[16] Miguel Lpez-Bentez and Fernando Casadevall, "Improved energy detection spectrum sensing for cognitive radio," Thsis paper published in IET communication publication, IET Communications(2012), 6(8):785.

[17] Saqib Saleem and Khurram Shahzad, "Performance evaluation of energy detection based spectrum sensing technique for wireless channel," International journal of multidisciplinary sciences and engineering, Vol. 3, no. 5, may 2012.

[18] Zhiqiang Bao, Bin Wu, Pin-Han Ho and Xiang Ling, "Adaptive threshold control for energy detection based spectrum sensing in cognitive radio networks," This full text paper was peer reviewed at the direction of IEEE Communications Society subject matter experts for publication in the IEEE Globecom 2011 proceedings.

[19] S.Shobana, R.Saravanan, R.Muthaiah, "Matched filter based spectrum sensing on cognitive radio for OFDM WLANs," International Journal of Engineering and Technology (IJET), Vol. 5 Issue 1, p142, February 2013. 